\title{
QUEEN'S
UNIVERSITY
BELFAST
}

\section{An analysis of the clinical appropriateness of out-of-hours emergency dental prescribing of antibiotics in Northern Ireland}

McKay, J., Begley, E., Kerlin, P., O'Carolan, D., Cleary, G., McKenna, G., \& Burnett, K. (2020). An analysis of the clinical appropriateness of out-of-hours emergency dental prescribing of antibiotics in Northern Ireland. British Dental Journal. https://doi.org/10.1038/s41415-020-1435-8

Published in:

British Dental Journal

Document Version:

Peer reviewed version

Queen's University Belfast - Research Portal:

Link to publication record in Queen's University Belfast Research Portal

Publisher rights

Copyright 2020 Springer Nature. This work is made available online in accordance with the publisher's policies. Please refer to any applicable terms of use of the publisher.

\section{General rights}

Copyright for the publications made accessible via the Queen's University Belfast Research Portal is retained by the author(s) and / or other copyright owners and it is a condition of accessing these publications that users recognise and abide by the legal requirements associated with these rights.

Take down policy

The Research Portal is Queen's institutional repository that provides access to Queen's research output. Every effort has been made to ensure that content in the Research Portal does not infringe any person's rights, or applicable UK laws. If you discover content in the Research Portal that you believe breaches copyright or violates any law, please contact openaccess@qub.ac.uk. 


\section{An analysis of the clinical appropriateness of out-of-hours emergency dental prescribing}

\section{of antibiotics in Northern Ireland}

\section{Authors:}

Jennifer McKay, School of Dentistry, Belfast Health and Social Care Trust

Edward Begley, School of Pharmacy and Pharmaceutical Sciences, Ulster University

Padraig Kerlin, School of Dentistry, Belfast Health and Social Care Trust

Donncha O'Carolan, Health and Social Care Board, Northern Ireland

Gerry Cleary, Health and Social Care Board, Northern Ireland

Gerry McKenna, Centre for Public Health, Queen's University Belfast

Kathryn Burnett, School of Pharmacy and Pharmaceutical Sciences, Ulster University 


\section{Abstract:}

The inappropriate prescribing of antibiotics in dentistry is potentially linked to the development of antimicrobial resistance, as well as being a considerable cost to healthcare. This study analysed the clinical appropriateness of antibiotics prescribed from 'walk-in' and telephone triage out-of-hours emergency dental clinics in Northern Ireland.

Methods: Patient and prescribing data were collected from two out-of-hour emergency dental clinics over a two month period between September and December 2017. In total 434 prescriptions were analysed. Clinical appropriateness was determined on a case-by-case basis for each prescription by referencing dental prescribing guidelines.

Results: Over half of the prescriptions analysed (52.77\%) were judged as clinically inappropriate. A total of $19.12 \%$ of prescriptions were judged as inappropriate as the antibiotic prescribed was not indicated for the diagnosis recorded by the clinician. Local measures were not attempted in $36.6 \%$ of cases. A significant difference $(p=0.002)$ was observed between the clinical appropriateness of prescriptions issued via walk-in and triage appointments with triage appointments issuing more clinically appropriate prescriptions.

Conclusions: A significant number of prescriptions provided in out-of-hours emergency dental clinics in Northern Ireland were judged to be inappropriate according to current dental prescribing guidelines. 


\section{Introduction:}

Antimicrobial resistance (AMR) poses a serious issue to today's society as it threatens the effective prevention and treatment of an ever-increasing range of infections. New resistance mechanisms are emerging and spreading globally, threatening our ability to treat common infectious diseases, which results in prolonged illness, disability, and death. It is predicted that by 2050, antimicrobial resistance will kill 10 million people a year and without effective antimicrobials, straightforward, everyday operations could be too dangerous to perform. ${ }^{1}$ It also increases the cost of health care with lengthier stays in hospitals and more intensive care required and it is predicted that by 2050 , the estimated global cost of antibiotic resistant infections will be $£ 44.7$ to $£ 74.5$ trillion. $^{2}$ The misuse and overuse of antimicrobials is accelerating the resistance process and has catalysed the explosion of antibiotic resistant strains of bacteria, rendering some infections very difficult to cure such as Methicillin-resistant Staphylococcus aureus (MRSA) or extremely drugresistant tuberculosis. ${ }^{2,3}$

Antimicrobial stewardship is key in combating AMR and embodies an organisational and system-wide approach to promoting and monitoring the judicious use of antimicrobials to preserve their future effectiveness. ${ }^{4,5}$ Antimicrobial stewardship encompasses areas such as monitoring and evaluating antimicrobial prescribing, providing regular feedback to prescribers, providing education and training to practitioners and integrating audit into existing quality improvement programmes. ${ }^{5} \mathrm{~A}$ key component is that prescribers should follow local or national guidance on prescribing, particularly around giving the most appropriate dose, the shortest effective course and the ideal route of administration to 
allow the best clinical outcomes for treatment of infection whilst minimising toxicity to the patient and subsequent resistance..$^{5,6}$ To try and control AMR, the government has recently published a 20-year vision and 5-year national action plan which explains how the United Kingdom will contribute to containing and controlling AMR by 2040. It includes targets such as reducing the use of antibiotics by $15 \%$ and cutting the number of drug resistant infections by $10 \%$ focusing on ensuring that current antibiotics remain effective. ${ }^{1}$

Studies have shown that inappropriate prescribing of antibiotics are having a significant impact on the development of AMR. ${ }^{7}$ An audit looking at antimicrobial prescribing by dentists in Wales saw that there was widespread use of antimicrobials managing acute dental conditions in the absence of signs of spreading infection and without adjunctive local measures, which increases patients' risk of developing adverse reactions to antimicrobial agents and places them at higher risk of experiencing a longer and more severe period of infection. ${ }^{8}$ In Northern Ireland dental prescriptions account for approximately $8 \%$ of antibiotics dispensed in primary care and combined with the fact that Northern Ireland prescribes the most antibiotics in the UK per capita, it is worthwhile investigating the clinical appropriateness of antibiotic dental prescriptions in the country. ${ }^{9,10}$

The aim of this quality improvement project was to determine the clinical appropriateness of antibiotic prescriptions issued from out-of-hours emergency dental clinics (OOH EDC) in Northern Ireland over a two month period. 


\section{Materials and methods:}

In Northern Ireland there are currently three out-of-hours emergency dental clinics which provide emergency dental care for patients. These facilities are geographically spread through Northern Ireland and are located at Belfast City Hospital (BCH), Dalriada Urgent Care Clinic (DUC) in Ballymena County Antrim and Craigavon Area Hospital in County Armagh. For the purposes of this study $\mathrm{BCH}$ and DUC were selected for inclusion as they receive the largest volume of patients. Additionally, they operate two different appointment systems with BCH providing a 'walk-in' service and DUC using a telephone triage service.

A data extraction sheet was created to record antibiotic prescribing data from both emergency dental clinics. The sheet was used to record the antibiotic prescribed, clinical diagnoses, prescription guideline used in determining appropriateness (if applicable) and factors which would influence prescription appropriateness for each case (Table 1). Further information on prescription appropriateness was collected on prescription non-complaint with guidelines, local measures not attempted, unnecessary prophylactic prescribing after local measures achieved, patient allergic to prescribed antibiotic and incomplete data.

A previous analysis of out-of-hours dental prescribing in Northern Ireland had found approximately 1200 antibiotic prescriptions issued from DUC and 1800 from BCH between December 2014 and May 2015. Extrapolating these figures, it was estimated that over two months in 2017, 400 and 600 prescriptions would be issued from DUC and BCH respectively. 
A sample size calculation based on the previous analysis indicated that 198 prescriptions from DUC and 236 from BCH would need to be analysed as part of the project. It was not necessary to obtain ethical approval from the Office for Research Ethics Committee for Northern Ireland as this research was a service evaluation. Approval was, however, obtained from Ulster University Biomedical Sciences Ethics Filter Committee and an honorary contract was obtained for the researcher with the Health and Social Care Board.

Fully anonymized data was entered electronically into the collection sheets by a researcher using an encrypted laptop and immediately backed up onto an encrypted storage device. To ensure secure storage data the contents of both were accessed by separate passwords known only by the lead researcher and project supervisor. During data collection there was input from lead dental clinicians to help interpret clinical notes and analyse the data to ensure it was valid from a dental perspective.

Following data collection all prescriptions were categorised as: appropriate, potentially inappropriate and inappropriate. Appropriate prescribing was defined as prescribing an antibiotic for which there was a clear clinical need and is suitable for the patient and their diagnosis according to prescribing guidelines. ${ }^{11}$ Potentially inappropriate was defined as antibiotic prescriptions which may be appropriate but appropriateness cannot be confirmed. This included cases of missing prescribing or patient data or cases in which the severity of the infection was not noted. ${ }^{12,13}$ In this study inappropriate prescribing was defined as: 
- $\quad$ prescribing an antibiotic for a patient in the absence of documented evidence of bacterial infection;

- $\quad$ prescribing a critical broad-spectrum antibiotic to patients in the absence of documented rationale;

- $\quad$ continuing an antibiotic prescription beyond the course length recommended in local or national guidelines. ${ }^{14}$

Clinical appropriateness of each prescription was determined by the lead researcher in conjunction with lead dental clinicians on a case-by-case basis using Scottish Dental Clinical Effectiveness Programme (SDCEP) antibiotic prescribing guidelines, Faculty of General Dental Practice UK (FGDP) antibiotic prescribing guidelines, British National Formulary (BNF) and British National Formulary for Children (BNFC) for reference.

'Prescription non-compliant with guidelines' was determined by looking at the guidelines for antibiotic regimes recommended for the patient's diagnosis and classifying as noncomplaint if the antibiotic, dose or duration did not correspond. 'Local measures not attempted' was confirmed by inspecting clinical notes. 'Incomplete data' included cases where information was omitted from the data collection form that are essential in determining appropriateness such as age, allergy status, strength, dose or duration. 'Unnecessary prophylactic prescribing after local measures achieved' was determined by assessing severity of the infection from clinical notes and 'patient allergic' related to cases 
where the patient had a clear recorded allergy in clinical notes yet was prescribed an antibiotic they were allergic to.

The completed data was transferred and interpreted using IMB ${ }^{\circledR}$ SPSS ${ }^{\circledR}$ Statistics 24 spreadsheet to give frequency statistics which were then used to show the overall trend of appropriate prescribing. Statistical testing was based on the null hypothesis which stated that that no antibiotic prescriptions provided during the study period would be judged as inappropriate.

\section{Results:}

A total of 434 antibiotic prescriptions were analysed during the course of this study: 198 from DUC and 236 from $\mathrm{BCH}$. Clinical appropriateness of the antibiotic prescriptions was determined by examining clinical records for the diagnosis, treatment carried out and adherence to guidelines on a case-by-case basis. Overall, it was found that a majority were inappropriate at $52.77 \%$, potentially inappropriate at $11.75 \%$, leaving only $35.48 \%$ appropriate.

There were significant differences observed in the numbers of appropriate prescriptions issued between the two study sites. It was demonstrated that DUC issued more clinically appropriate prescriptions compared to $\mathrm{BCH}(\mathrm{p}=0.002$ Pearson Chi-Square test) (Figure 1). Significant differences were observed between levels of appropriate prescribing according 
to consultation type with the largest proportion of appropriate prescriptions issued after a telephone triage at DUC (60.27\%), followed by a clinical appointment at DUC (42.4\%) and walk-in appointments at $\mathrm{BCH}(25.42 \%)$.

The data did not reveal any significant differences observed between patient gender ( $p=0.815$ Pearson Chi-Square test). For patients over the age of $80,100 \%$ of prescriptions were judged as inappropriate and patients aged $10-19$ years, $64.6 \%$ were judged as inappropriate. The age group with the greatest levels of appropriate prescribing was 20-29 years with $58.6 \%$ appropriate prescribing noted (Figure 2).

Dentoalveolar abscess, irreversible pulpitis and reversible pulpitis accounted for $67-100 \%$ of clinical diagnoses in all age groups. Pericoronitis was most prevalent in patients between 20 and 29 , accounting for $23.57 \%$ of the diagnoses in this age group. The levels of appropriate prescribing were highest with $76.5 \%$ in the most frequently presenting conditionsdentoalveolar abscess and pericoronitis (Figure 3). There was significance observed between the patient's diagnosis and the clinical appropriateness of antibiotic prescriptions issued at both clinics. The highest levels of inappropriate prescribing were seen in the following conditions: dry socket, reversible pulpitis, irreversible pulpitis and broken teeth which totalled $19.12 \%$ of cases. No significance was observed between the antibiotic prescribed and cases where local measures were not attempted $(p=0.543$ Pearson ChiSquare test), nor was there any significance between the antibiotic prescribed and cases in which the antibiotic was prescribed for prophylaxis after local measures were achieved 
$(p=0.981$ Pearson Chi-Square test). Two cases were observed at $\mathrm{BCH}$ in which the patient was allergic to the antibiotic prescribed.

Significance was observed between the antibiotic prescribed and cases in which the prescription was non-compliant with prescribing guidelines $(p<0.001$ Pearson Chi-Square test). This was the only factor identified in which the type of antibiotic prescribed had a direct and significant impact on the clinical appropriateness of the prescription. Prescriptions which had dosages and durations recorded, and clear associated diagnoses, were grouped by which guidelines they complied with. This selection accounted for 287 prescriptions, $66.13 \%$ of the total sample. The largest proportion of prescriptions in this category were those that complied with all three guidelines, followed by those that complied with FGDP(UK) and BNF/BNFC guidelines only, and then those that complied with BNF/BNFC guidance only. There were no prescriptions that complied with the SDCEP and FGDP(UK) guidelines only. It is apparent that most prescriptions complied with the BNF/BNFC guidance (Figure 4). No significance was observed between the guidelines a prescription complied with and the clinical appropriateness of the prescription $(p=0.077$ Pearson Chi-Square test), but significance was observed between the guidelines that the antibiotic prescriptions complied with and the clinic attended $(p=0.005$ Pearson Chi-Square test). Both clinics mainly prescribed in accordance with all three guidelines, with DUC prescribing more frequently in accordance with BNF/BNFC guidelines only. 


\section{Discussion:}

The aim of this project was to explore levels of inappropriate prescribing within two EDCs in Northern Ireland. A total of $52.77 \%$ of antibiotic prescriptions were judged to be inappropriate during the timeframe of this project. Additionally, a further $11.75 \%$ of prescriptions were potentially inappropriate. These results can be compared to a study which showed that antibiotics were inappropriately prescribed in three quarters of patients across 5 EDCs in East England. ${ }^{13}$ More recently, in a cross-sectional study of GDP antibiotic prescribing in Wales, $81 \%$ of antibiotics were prescribed inappropriately. ${ }^{15}$ In comparison to these studies, the level of inappropriate antibiotic prescribing observed at DUC and $\mathrm{BCH}$ emergency dental clinics is relatively low. However, a target standard of $0 \%$ inappropriate antibiotic prescribing is essential to combat AMR effectively. ${ }^{7,16}$

This is the first known study to compare the appropriateness of antibiotic prescriptions from triage and non-triage based out-of-hours emergency dental clinics. The clinical appropriateness of prescriptions varies significantly between DUC and $\mathrm{BCH}$ and shows that the DUC service model, which includes a triage system, is issuing more clinically appropriate antibiotic prescriptions. The walk-in nature of $\mathrm{BCH}$ EDC may be putting considerable time pressures on GDPs and impacting their clinical decision making and quality of patient care.

The largest age group of patients who attended for out of hours management were those between 20 and 29. It has been shown that sugar consumption in the UK is highest between the ages of 4 and $18 .{ }^{17}$ The large sugar intake at these ages causes the development of 
caries which if left untreated can progress to dentoalveolar abscess in later years which may explain the large numbers of patients between 20 and 29 that attended the emergency dental clinics with conditions like irreversible pulpitis and abscesses. Another reason for these large figures may be due to the eruption of the third molars, which commonly occurs in the late teens and twenties. The eruption of these teeth can cause pericoronitis, which was the second most common reason for antibiotic prescribing in this study. ${ }^{18}$

There was a significant difference between the clinical appropriateness of the antibiotic prescribed and the patient diagnosis as observed at both clinics. This difference is likely due to the prescribing for conditions that have no indication for antibiotics which accounted for 19.12\% of all prescriptions analysed. Guidelines state that there is no evidence to support the use of antibiotics for the treatment of pulpitis or dry socket, as these conditions are inflammatory in nature and can be effectively and fully treated with local measures alone such as extirpation or irrigation. They also state that local measures should always be carried out as a first line treatment where possible, and that antibiotics should only be used when there are signs of systemic involvement, severe infection, or when local measures are not possible. ${ }^{19,20}$ As local measures were not possible for DUC triage patients, because these patients did not attend the clinic, there was a greater likelihood that prescriptions issued via triage would be appropriate. Excluding the above cases, it was found that local measures were not attempted $36.6 \%$ of the time. Compared with a cross-sectional study analysing antibiotic prescribing by GDPs in Wales, it was found that antibiotics were prescribed without local measures being attempted $70.6 \%$ of the time. ${ }^{15}$ 
Patient allergy was recorded significantly less at DUC compared to $\mathrm{BCH}$ and this may be explained by the fact that BCH EDC has an 'allergies questionnaire' sheet attached to all patient records. This sheet serves as a prompt for GDPs to check for patient allergies in every consultation. Evidently, it is an effective way of encouraging GDPs to record patient allergies. It was observed that antibiotic dosages and durations were recorded significantly less at $\mathrm{BCH}$. This may be explained by the 'walk-in' nature of $\mathrm{BCH}$ as it is more stressful and there is less time during patient consultations, resulting in a poorer quality of record keeping. Two cases were recorded at $\mathrm{BCH}$ in which the patient had a penicillin allergy, yet they were prescribed amoxicillin, which is a serious prescribing error as the patients may have had a potentially life-threatening anaphylactic reaction. This may have occurred due to $\mathrm{BCH}$ being substantially busier than DUC and the triage system in place at DUC was designed to take time pressures off GDPs reducing the likelihood of errors like this.

During this study, it was discovered that there are inconsistencies between the prescribing guidelines regarding the dosages and durations of antibiotics (Table 2). Clearly there is a need for greater consistency between all guidelines. It is seen that the BNF/BNFC is the most commonly used prescribing guidelines by GDPs at these clinics. The BNF/BNFC is the most definitive and up-to-date source for drug prescribing in the UK, which may explain why it is the most frequently used. It is possible that GDPs are more familiar with the BNF/BNFC than the other guidelines, as all GDPs in Northern Ireland are issued a copy every year by the Business Service Organisation. The implementation of antibiotic prescribing guidelines is linked with reduced rates of patient harm from prescribing errors, a reduction in AMR and an increase in the appropriate prescribing of antibiotics. ${ }^{21}$ Hence, it is vitally important that 
GDPs at these EDCs have clear, coherent and up-to-date guidelines on the prescribing of antibiotics.

This project is subject to several limitations. The first is that clinical appropriateness of each prescription was determined by the researcher and lead dental clinicians using patient records to determine if it complied with guidelines. This is wholly dependent on the quality of notes recorded and in the busier 'walk-in' service at $\mathrm{BCH}$ it has been demonstrated that they are subject to more substantial time pressures which could impact quality of care provided and record keeping. A potential way to alleviate this issue would be to provide a proforma for use in $\mathrm{BCH}$, which would serve as a prompt for GDPs to ensure key information is not forgotten. Another limitation is that it was only carried out in two out-of-hours services so the overall sample size is small which means that it might not be representative of out-of-hours services in general. Ideally in the future, further evaluation of these services would include more sites to allow results to be more precise. A number of patients who attend out-of-hours services are not registered with a GDP, citing dental fear as a major factor for this. When they do attend these services, not all consent for local measures to be carried out to remove the source of pain or infection which leaves the treating dentists few options other than to prescribe an antibiotic when it may not be indicated. This raises the question whether these results are generalisable to wider prescribing practices. 


\section{Conclusion:}

This is the first study to have analysed the clinical appropriateness of antibiotics prescribed from EDCs in Northern Ireland. It was found that the majority of antibiotics were not prescribed appropriately from these clinics during the study period. A major reason for this is due to the prescribing of antibiotics for conditions which antibiotics are not indicated. While localised infection may be present with these conditions, it is recommended that local measures should always be attempted as a first line treatment when possible as they are often the most effective treatment for removing the source of the infection. This study found that local measures were attempted in $63.4 \%$ of patients who received an antibiotic prescription.

It was discovered that the DUC issued more clinically appropriate prescriptions than $\mathrm{BCH}$. It is proposed that the differences in service model design are main reason for this as it may relieve time pressures on GDPs. Another notable finding from this study is that there is significant variation between the prescribing recommendations in the antibiotic prescribing guidelines for dental practice.

Recommendations from this study:

- Launching a campaign across Northern Ireland to refresh GDPs knowledge that antibiotics should not be prescribed for conditions with only localised infection present with an emphasis on the issue of AMR. 
- $\quad$ Local measures encouraged more at the EDCs.

- Commissioners of the EDCs review the service model at $\mathrm{BCH}$, and consider implementing DUC's service model as this may reduce prescribing errors, improve record keeping and improve patient care.

- Introduce a standardised, systematic approach of recording allergies at DUC, similar to $\mathrm{BCH}$.

- Department of Health reviews the guidelines currently in use, and provides updated advice to all GDPs in Northern Ireland on which guidelines to follow for antibiotic prescribing, considering the highlighted inconsistencies.

The progression of AMR is certainly posing a significant threat to global public health, and the inappropriate prescribing of antibiotics is prevalent in all areas of healthcare. ${ }^{3}$ However, with the findings from this study and the above recommendations, the dental profession in Northern Ireland can make a significant impact on the global battle currently happening against AMR.

\section{Declaration of interests:}

There are no conflicts of interest. 


\section{References:}

1. Gov UK. Antimicrobial resistance: UK launches 5-year action plan and 20-year vision. 2019. Online information available at https://www.gov.uk/government/news/antimicrobialresistance-uk-launches-5-year-action-plan-and-20-year-vision (accessed June 2019).

2. Wellcome Trust and UK Government. Tackling drug-resistant infections globally: final report and recommendations. 2016. Online information available at https://amrreview.org/sites/default/files/160525_Final\%20paper_with\%20cover.pdf (accessed June 2019).

3. World Health Organisation. Antimicrobial resistance. 2018. Online information available at https://www.who.int/en/news-room/fact-sheets/detail/antimicrobialresistance (accessed April 2019).

4. Department of Health Northern Ireland. Strategy for Tackling Antimicrobial Resistance. 2013. Online information available at https://www.healthni.gov.uk/sites/default/files/publications/dhssps/arac-strategy-for-tackling-antimicrobialresistance-star-2012-17.pdf (accessed April 2019).

5. National Institute for Health and Care Excellence. Antimicrobial stewardship: systems and processes for effective antimicrobial medicine use. 2015. Online information 
available at https://www.nice.org.uk/guidance/ng15/resources/antimicrobial-stewardshipsystems-and-processes-for-effective-antimicrobial-medicine-use-pdf-1837273110469 (accessed June 2019).

6. Doron S, Davidson LE. Antimicrobial Stewardship. Mayo Clinical Proceedings 2011 Nov; 86: 1113-1123

7. Sweeney LC, Dave J, Chamber PA, Heritage J. Antibiotic resistance in general dental practice- a cause for concern? Journal of Antimicrobial Chemotherapy 2004; 53: 567-576.

8. Cope Al, Barnes Em Howells EP et al. Antimicrobial prescribing by dentists in Wales, UK: findings of the first cycle of clinical audit. British Dental Journal 2016; 221: 25-30.

9. Health and Social Care information Centre. 2015.

10. Department of Health N.Ireland. Chief Medical Officer Annual Report 2016-17. 2017. Online information available at https://www.healthni.gov.uk/sites/default/files/publications/health/CMO\%20Annual\%20Report\%20201617_0.pdf (accessed April 2019). 
11. Buetow SA, Sibbald B, Cantrill JA, Halliwell S. Appropriateness in healthcare: application to prescribing. Social Science and Medicine 1997; 45: 261-271.

12. Baclet N, Ficheur G, Alfandari S et al. Explicit definitions of potentially inappropriate prescriptions of antibiotics in older patients: a complication derived from systematic review. International Journal of Antimicrobial Agents 2017; 50: 640-648.

13. Daley YM, Martin MV. Are antibiotics being used appropriately for emergency dental treatment? British Dental Journal 2001; 191: 391-393.

14. Advisory Committee on Antimicrobial Prescribing, Resistance and Healthcare Associated Infection (APRHA). Annual Report. 2017. Online information available at https://assets.publishing.service.gov.uk/government/uploads/system/uploads/attachment_ data/file/714000/APRHAI_Annual_Report_2016-2017_.pdf (accessed June 2019).

15. Cope AL, Francis NA, Wood F, Chestnut IG. Antibiotic prescribing in UK general dental practice: a cross-sectional study. Community Dentistry and Oral Epidemiology 2016; 44: $145-153$ 
16. Palmer NOA. Antimicrobial resistance and antibiotic prescribing in dental practice. Dental Update 2016; 45: 954-960

17. Public Health England. Sugar reduction: the evidence for action. 2015. Online information available at https://assets.publishing.service.gov.uk/government/uploads/system/uploads/attachment_ data/file/470179/Sugar_reduction_The_evidence_for_action.pdf (accessed April 2019).

18. Mitchell DA, Mitchell L, McCaul L. Oxford handbook of clinical dentistry. 6th ed. Oxford: Oxford University Press, 2014.

19. Faculty of General Dental Practice. Antimicrobial prescribing standards. 2nd ed. 2014. Online information available at https://www.fgdp.org.uk/antimicrobial-prescribingstandards (accessed April 2019).

20. Scottish Dental Clinical Effectiveness Programme. Drug Prescribing for Dentistry. 3rd ed. 2016. Online information available at http://www.sdcep.org.uk/wpcontent/uploads/2016/03/SDCEP-Drug-Prescribing-for-Dentistry-3rd-edition.pdf (accessed April 2019) 
21. Schuts EC, Hulscher MEJL, Mouton JW et al. Current evidence on hospital antimicrobial stewardship objectives: a systematic review and meta-analysis. The Lancet Infectious Diseases 2016; 16: 847-856. 